\title{
Kinetics of Suicide Substrates
}

\author{
Stephen G. WALEY \\ Sir William Dunn School of Pathology, University of Oxford, South Parks Road, \\ Oxford OX1 3RE, U.K.
}

(Received 6 November 1979)

\begin{abstract}
When suicide substrates inactivate enzymes during catalysis, formation of product and inactivation of enzyme proceed concurrently. The steady-state hypothesis is applicable when catalytic quantities of enzyme are used. Equations for the rate of inactivation have been derived and integrated to obtain equations describing progress curves.
\end{abstract}

The specific inactivation of enzymes often lies at the root of effective drug action, and it is, moreover, an important step in the study of the enzyme's active site. Certain substrates (suicide substrates, mechanism-based inactivators) undergo a structural change during the course of catalysis that results in their being bound to the enzyme and inactivating it (Endo et al., 1970; Abeles \& Maycock, 1976; Rando, 1977; Walsh et al., 1978; Seiler et al., 1978). It is characteristic of suicide substrates that formation of product and inactivation of enzyme proceed concurrently. The ratio of the rates of these processes (the partition ratio) remains constant throughout the reaction when catalytic amounts of enzyme are used. It is also commonly found that the inactivation is first-order, and that the observed rate constant varies hyperbolically with the concentration of the substrate (see, e.g., Meloche et al., 1972; Wang \& Walsh, 1978; Soper \& Manning, 1978). The observed kinetics are believed to follow from a scheme of the type (Walsh et al., 1978):

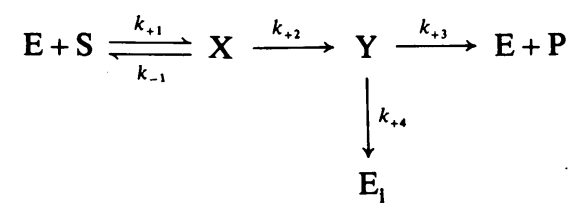

where $E, S$ and $P$ stand for enzyme, substrate and product respectively, and $X$ is the first intermediate (the enzyme-substrate complex) and $Y$ is another intermediate (e.g. an acyl-enzyme), and $E_{1}$ is inactivated enzyme. However, the kinetics that follow from this scheme do not seem to have been worked out. The inactivation of the enzyme is here taken to be irreversible over the time scale of the experiment.

\section{Steady-State Kinetics}

The following justification of the steady-state hypothesis is based on the treatment of simple enzymic reactions by Heineken et al. (1967). The concentrations of the species being denoted by italic lower-case symbols, the rate equations are:

$$
\begin{aligned}
& \frac{\mathrm{d} s}{\mathrm{~d} t}=-k_{+1} e \cdot s+k_{-1} x \\
& \frac{\mathrm{d} x}{\mathrm{~d} t}=k_{+1} e \cdot s-\left(k_{-1}+k_{+2}\right) x \\
& \frac{\mathrm{d} y}{\mathrm{~d} t}=k_{+2} x-\left(k_{+3}+k_{+4}\right) y
\end{aligned}
$$

Now the equations are made dimensionless by the following substitutions $\left(e_{0}\right.$ and $s_{0}$ being the initial values): $\quad w=s / s_{0}, \quad u=x / e_{0}, \quad v=y / e_{0}, \quad T=$ $k_{+1} e_{0} t, m=\left(k_{-1}+k_{+2}\right) / k_{+1} s_{0}, g=\left(k_{+3}+k_{+4}\right) / k_{+1} s_{0}$, $h=k_{+2} / k_{+1} s_{0}, n=k_{-1} / k_{+1} s_{0}, \quad z=e_{1} / e_{0}$. Hence (as $\left.e+x+y+e_{1}=e_{0}\right)$ :

$$
\begin{aligned}
& \frac{\mathrm{d} w}{\mathrm{~d} T}=-w[1-(u+v+z)]+n \cdot u \\
& \frac{e_{0}}{s_{0}} \cdot \frac{\mathrm{d} u}{\mathrm{~d} T}=w[1-(u+v+z)]-m \cdot u \\
& \frac{e_{0}}{s_{0}} \cdot \frac{\mathrm{d} y}{\mathrm{~d} T}=h \cdot u-g \cdot v
\end{aligned}
$$

When catalytic quantities of enzyme are used, $e_{0} / s_{0}$ is small, and the left-hand sides of eqns. (2) and (3) may be equated to zero. The equations may then be solved, as set out in what follows.

\section{Rate of Inactivation}

When inactivation is followed by withdrawing small portions from the incubation mixture of enzyme and suicide substrate at various times and adding these to an assay mixture containing a normal substrate, then the species $\mathrm{X}$ and $\mathrm{Y}$ will usually be converted into $\mathrm{E}$. Thus the activity $(a)$ is given by: $a=e_{0}-e_{1}$, and $-\mathrm{d} a / \mathrm{d} t=\mathrm{d} e_{1} / \mathrm{d} t=k_{+4} y$. This equa- 
tion may be written in terms of the dimensionless variables defined above:

$$
k_{+1} e_{0}<\frac{\mathrm{d} z}{\mathrm{~d} T}=k_{+4} v
$$

From eqns. (2) and (3):

and hence:

$$
v=k_{+4} \frac{h}{g} \cdot \frac{w(1-z)}{m+w(1+h / g)}
$$

$$
\frac{1}{1-z} \cdot \frac{\mathrm{d} z}{\mathrm{~d} T}=\frac{k_{+4}}{k_{+1} e_{0}} \cdot \frac{h}{g} \cdot \frac{w}{m+w(1+h / g)}
$$

Eq. (4) is now converted back into the conventional symbols:

$$
-\frac{\mathrm{d} \ln a}{\mathrm{~d} t}=\frac{A \cdot s}{B+s}
$$

where

and

$$
A=k_{+2} k_{+4} /\left(k_{+2}+k_{+3}+k_{+4}\right)
$$

$$
B=\left(\frac{k_{-1}+k_{+2}}{k_{+1}}\right)\left(\frac{k_{+3}+k_{+4}}{k_{+2}+k_{+3}+k_{+4}}\right)
$$

Eqn. (5) shows that the inactivation is not strictly first-order. In practice the deviation from linearity may not be apparent in the usual semilogarithmic plot (Fig. 1). The extent of the deviation depends on how much the concentration of substrate alters; the alteration will be small if $s_{0}>k_{+3} / k_{+4} e_{0}$. The apparent rate constant for inactivation will approximate to $A s_{0} /\left(B+s_{0}\right)$, which varies hyperbolically with $s_{0}$. The expressions for the parameters will of course be simplified if one of the steps is cleanly rate-limiting.

The calculated rate of product formation can be found from: $\mathrm{d} p / \mathrm{d} t=(r) \mathrm{d} e_{1} / \mathrm{d} t$, where $r=k_{+3} / k_{+4}$ (the partition ratio), or from eqn. (6) (which can be derived by straightforward manipulations):

$$
\frac{\mathrm{d} p}{\mathrm{~d} t}=\frac{A r e_{0} s_{0}\left(1-\frac{p}{r e_{0}}\right)}{s_{0}+\frac{B}{1-\frac{p}{s_{0}}}}
$$

The initial rate of formation of product $(p \rightarrow 0$ in eqn. 6) varies hyperbolically with the concentration of substrate.

\section{Progress Curves}

Since $\mathrm{d} a / \mathrm{d} t=(1 / r) d s / d t$, eqn. (5) may be written as:

$$
-\frac{1 \mathrm{~d} s}{A \mathrm{~d} t}=\frac{r \cdot a \cdot s}{B+s}
$$

and since $a=e_{0}-\left(s_{0}-s\right) / r$ :

$$
-\frac{1}{A} \cdot \frac{\mathrm{d} s}{\mathrm{~d} t}=\frac{s\left(e_{0} r-s_{0}+s\right)}{B+s}
$$
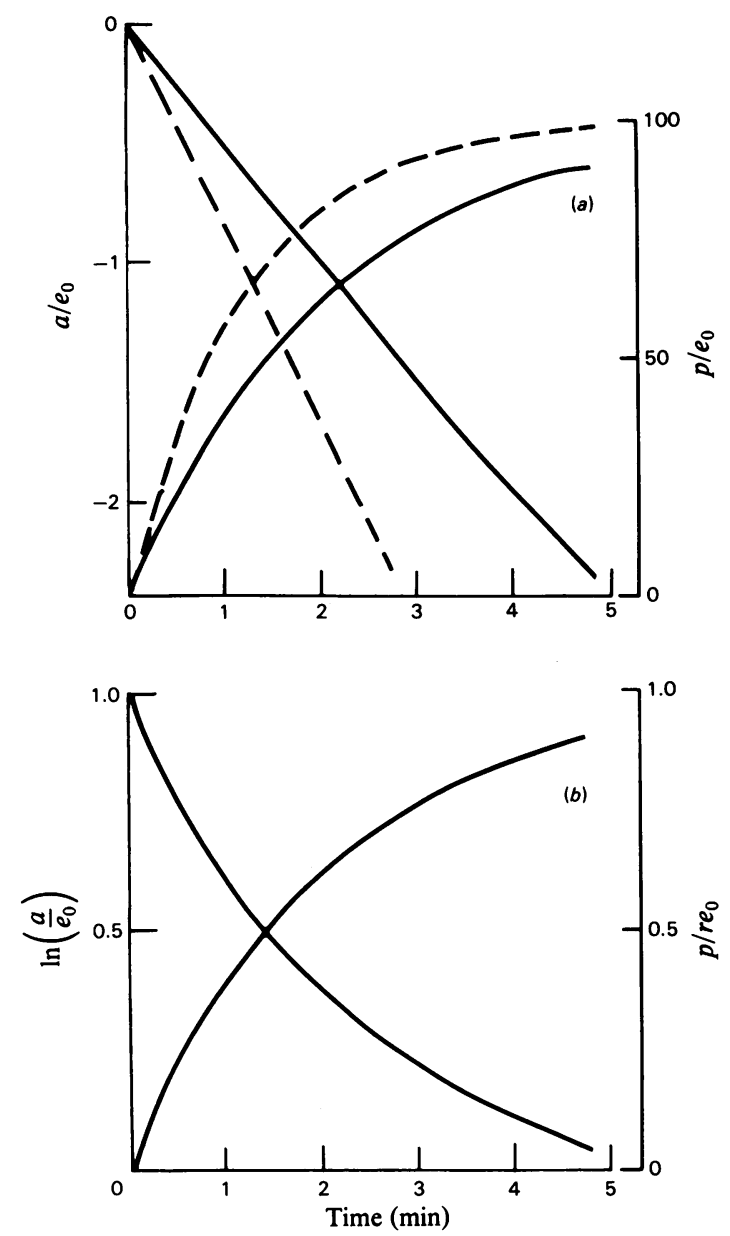

Fig. 1. Progress curves of loss of enzymic activity and formation of product

(a) The descending curves show the logarithm of the fractional activity, and the ascending ones show the concentration of the product as a fraction of the initial concentration of enzyme $\left(p / e_{0}\right)$; the final value of $p / e_{0}$ gives the partition ratio $\left(r=k_{+3} / k_{+4}\right.$ in the scheme in the text). The broken line shows the higher concentration of substrate $\left(s_{0}\right)$; note that $r$ is unaffected. (b) Progress curves showing the relation between the loss in fractional activity (descending curve) and the concentration of product as a fraction of its final value. - $s_{0} / e_{0}=1000 ; r=100$; $s_{0} / B=1 ; A=1 \mathrm{~min}^{-1}$. -,$- s_{0} / e_{0}=5000 ; r=100 ;$ $s_{0} / B=5 ; A=1 \mathrm{~min}^{-1}$. The lines are drawn by the use of eqns. (7) and (8) in the text, where $B$ is defined. 
Integration then gives:

$$
t=\frac{1}{A}\left\{\frac{B}{s_{0}-r e_{0}} \ln \left(\frac{1-p^{\prime}}{1-\mathrm{F} p^{\prime}}\right)-\ln \left(1-F p^{\prime}\right)\right\}
$$

where $F=s_{0} / r e_{0}$ and $p^{\prime}=p / s_{0}$. An example of a progress curve for product is given in Fig. 1. The fraction of enzymic activity remaining can either be found from: $a / e_{0}=1-F p^{\prime}$, or from eqn. (8):

$$
t=\frac{1}{A}\left(\frac{B}{s_{0}-r e_{0}} \ln \left\{\frac{e_{0}}{a}\left[1-\frac{r}{s_{0}}\left(e_{0}-a\right)\right]\right\}-\ln \left(\frac{a}{e_{0}}\right)\right)
$$

The progress curve for inactivation is the reflection about the $50 \%$ conversion line parallel to the time axis of the progress curve for product formation, when they are scaled appropriately (Fig. 1). The half-life for either process is:

$$
t_{\frac{1}{2}}=\frac{1}{A}\left[\frac{\left(\frac{B}{s_{0}}\right) \ln \left(2-\frac{r e_{0}}{s_{0}}\right)}{1-\frac{r e_{0}}{s_{0}}}+\ln 2\right]
$$

\section{Effectiveness of Suicide Substrates}

Eqn. (9) is important because it shows the factors necessary for an effective suicide substrate. There are three parameters. The first, $A$, is effectively the $k_{\text {cat. }}$ for overall flux. Although the substrate has to be processed at a reasonable rate, the value of $A$ may be very much less than the $k_{\text {cat. }}$ for a good substrate without inactivation being inconveniently slow. This is why the usual structure-activity relationships for substrates are such poor guides for designing suicide substrates. The second parameter, $B / s_{0}$, is a measure of the fraction of enzyme that is uncombined; at high concentrations of substrate $s_{0} \gg B$, and then $t_{\frac{1}{2}}$ approaches $(1 / A) \ln 2$. Finally, the characteristic parameter of suicide substrates is $r e_{0} / s_{0}$, in other words the magnitude of the partition ratio compared -with the ratio of initial concentration of substrate to that of enzyme. In practice, it will sometimes be possible to compensate for an unfavourable partition ratio by a high value for $s_{0} / e_{0}$. Conversely, a favourable partition ratio permits a wider choice of values for $s_{0} / e_{0}$. Eqn. (9) should help in predicting the outcome of experiments in vivo from those in vitro. If $r e \ll s_{0}$, eqn. (9) reduces to eqn. (10).

$$
t_{\frac{1}{2}}=\frac{1}{A}\left[\ln 2\left(1+\frac{B}{s_{0}}\right)\right]
$$

\section{Estimation of the Parameters}

There are two ways in which a reaction obeying the mechanism in the scheme may come to an end. Usually, all the enzyme is inactivated, with only some of the substrate having been converted into product; this corresponds to $\left(s_{0} / e_{0}\right)>r$. The alternative is that all the substrate has been consumed and only some of the enzyme has been inactivated; here $\left(s_{0} / e_{0}\right)<r$. The first tests with a new suicide substrate should be devoted to distinguishing between these alternatives, by seeing which components are present when the reaction has come to a halt. For the rest of this discussion we assume that all the enzyme has been inactivated. The amount of product finally formed $\left(p_{\infty}\right)$ should be measured, when the concentration of the substrate is varied. The partition ratio, $r$, is then given by $p_{\infty} / e_{0}$ (Fig. 1); this gives the number of catalytic turnovers required to inactivate the enzyme. The partition ratio is independent of the concentration of the substrate, when the scheme applies.

The initial rate of inactivation may be measured at various concentrations of substrate, and eqn. (5) used to obtain $A$ and $B$. Preferably the direct linear plot should be used (Cornish-Bowden \& Eisenthal, 1978; Nimmo \& Atkins, 1979). Alternatively, the half-life for inactivation may be measured, and eqn. (9) used (an obvious extension of the direct linear plot may be used here too).

The support of the Medical Research Council is gratefully acknowledged; this paper is a contribution from the Oxford Enzyme Group.

\section{References}

Abeles, R. H. \& Maycock, A. L. (1976) Acc. Chem. Res. 9, 313-319

Cornish-Bowden, A. \& Eisenthal, R. (1978) Biochim. Biophys. Acta 523, 268-272

Endo, K., HelmKamp, G. M. \& Bloch, K. (1970) J. Biol. Chem. 245, 4293-4296

Heineken, F. G., Tsuchiya, H. M. \& Aris, R. (1967) Math. Biosci. 1, 95-113

Meloche, H. P., Luczak, M. A. \& Wurster, J. M. (1972) J. Biol. Chem. 247, 4186-4191

Nimmo, I. A. \& Atkins, G. L. (1979) Trends Biochem. Sci. 4, 236-238

Rando, R. R. (1977) Methods Enzymol. 46, 28-41

Seiler, N., Jung, M. J. \& Koch-Weser, J. (1978) Enzyme-activated Irreversible Inhibitors, Elsevier/ North-Holland Biomedical Press, Amsterdam

Soper, T. S. \& Manning, J. M. (1978) Biochemistry 17, 3377-3384

Walsh, C., Cromartie, T., Mariotte, P. \& Spencer, R. (1978) Methods Enzymol. 53, 437-448

Wang, E. \& Walsh, C. (1978) Biochemistry 17, 13131321 